\title{
Inhibition of the NADPH Oxidase Pathway Reduces Ferroptosis during Septic Renal Injury in Diabetic Mice
}

\author{
Weifeng Yao $\mathbb{D}^{1},{ }^{1}$ Haofeng Liao, ${ }^{1}$ Mengya Pang, ${ }^{1}$ Lijie Pan, ${ }^{2}$ Yu Guan, ${ }^{1}$ Xiaolei Huang, ${ }^{3}$ \\ Ziqing Hei, ${ }^{1}$ Chenfang Luo, ${ }^{1}$ and Mian Ge $\left(\mathbb{i}^{1}{ }^{1}\right.$ \\ ${ }^{1}$ Department of Anesthesiology, Third Affiliated Hospital, Sun Yat-sen University, Guangzhou, Guangdong 510630, China \\ ${ }^{2}$ Vaccine Research Institute of Sun Yat-sen University, Guangzhou 510630, China \\ ${ }^{3}$ Department of Anesthesiology, Shenzhen Maternity and Child Healthcare Hospital, Southern Medical University, \\ Shenzhen 518116, China
}

Correspondence should be addressed to Mian Ge; gemiansums@163.com

Received 30 June 2021; Revised 25 January 2022; Accepted 3 February 2022; Published 27 February 2022

Academic Editor: Liang-Jun Yan

Copyright (C) 2022 Weifeng Yao et al. This is an open access article distributed under the Creative Commons Attribution License, which permits unrestricted use, distribution, and reproduction in any medium, provided the original work is properly cited.

Background. Obesity and type 2 diabetes mellitus (DM) contribute to a higher mortality rate in patients with septic acute kidney injury (AKI) during sepsis. Reactive oxygen species (ROS) is the major injury factor for sepsis. This study was aimed at exploring the potential therapeutic drug for septic AKI targeting on ROS. Methods. A murine septic AKI model was established in both wildtype and high-fat diet-fed (HFD) mice. NADPH oxidase inhibitor Vas2870 was used in vivo to explore the role of NADPH oxidase in ROS release in septic AKI in diabetic mice. Ferrostatin-1 was administered to investigate the role of ferroptosis in ROS accumulation during NADPH oxidase activating in septic AKI in diabetic mice. Results. Compared to chow diet-fed mice, HFD diabetic mice which were subjected to LPS exhibited aggravated renal function (blood urea nitrogen, creatinine clearance, and serum cystatin C) and oxidative stress (malondialdehyde, 4-HNE, ROS, 8-OHdG, and NADPH oxidase), thus resulting in a higher mortality rate. Septic renal injury was significantly attenuated by the ferroptosis inhibitor Fer-1 in HFD-challenged mice. Furthermore, ferroptosis accumulation and related protein expression (ASCL4, FTH1, and GPX4) were altered by LPS stimulation in HFD-challenged mice and suppressed by NADPH oxidase inhibition via Vas2870 in vivo. In summary, NADPH inhibition restored septic renal function from injury by suppressing ferroptosis accumulation in HFD-challenged mice. Conclusion. These results suggest that targeting NADPH-mediated ROS release and ferroptosis accumulation is a novel therapeutic strategy to protect the kidney from septic injury in patients with obesity and type $2 \mathrm{DM}$.

\section{Introduction}

Diabetes mellitus $(\mathrm{DM})$ is a prevalent global health problem that is one of the fastest growing emergencies of the 21st century, with approximately $10 \%$ morbidity [1]. Sepsis, another critical global medical condition, presents a mortality rate of approximately $26 \%$ [2]. Moreover, a recent study showed that approximately $21.8 \%$ of patients with sepsis had a medical history of DM [3]. Patients with DM show increased morbidity and mortality from sepsis compared to those in the nondiabetic population [4].

Acute kidney injury (AKI) is a common complication of sepsis, with a morbidity rate of $40 \%$ [5]. Although Esper et al. showed that diabetic patients were less likely to develop acute respiratory failure and hematological dysfunction than those without diabetes [6], recent studies indicate that diabetes is an independent risk factor for postoperative infection and sepsis-associated AKI [6, 7]. Sepsis-associated AKI results in higher mortality $(20 \%-60 \%)$ compared with that of other types of AKI [8]. Reducing sepsis-associated AKI and restoring renal function can improve prognosis, particularly in patients with DM [9].

The pathogenesis of sepsis-associated AKI is poorly understood, and several factors, including systemic hemodynamics, renal microcirculation disturbance, and renal hypoxia, may be involved [10]. The production of reactive oxygen species (ROS) from the kidneys or other organs can directly damage renal parenchyma and may aggravate 


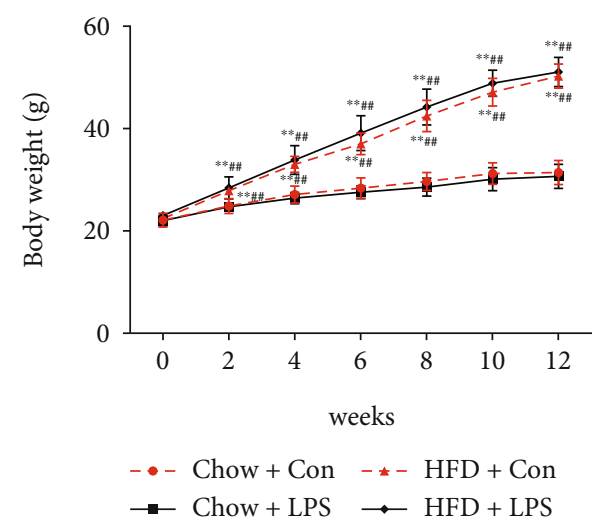

(a)
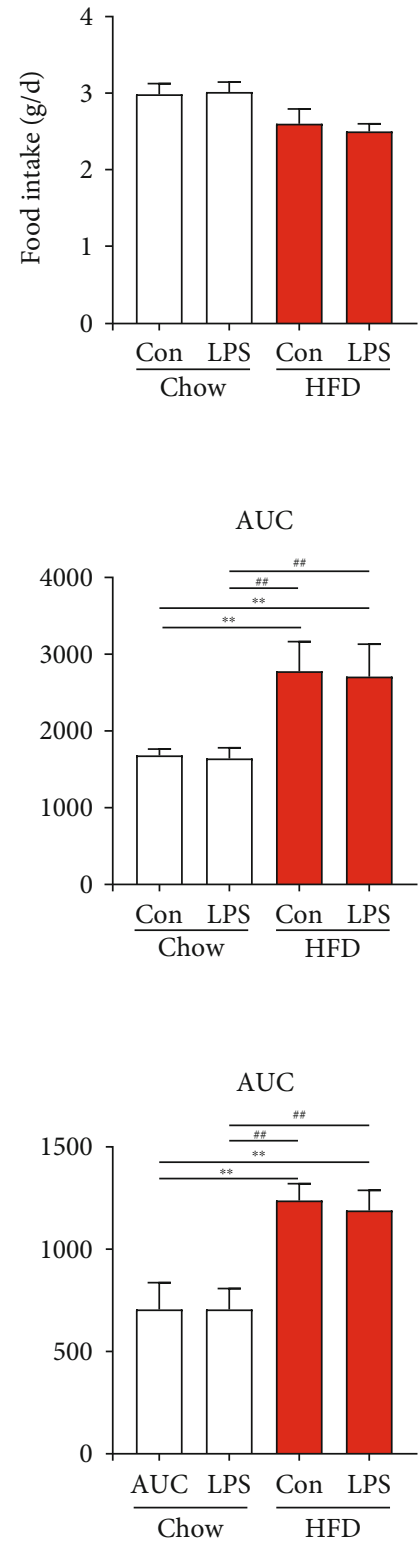

(b)

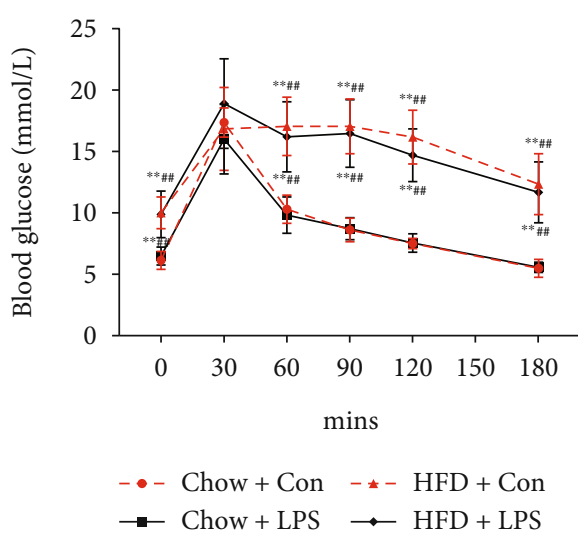

(c)

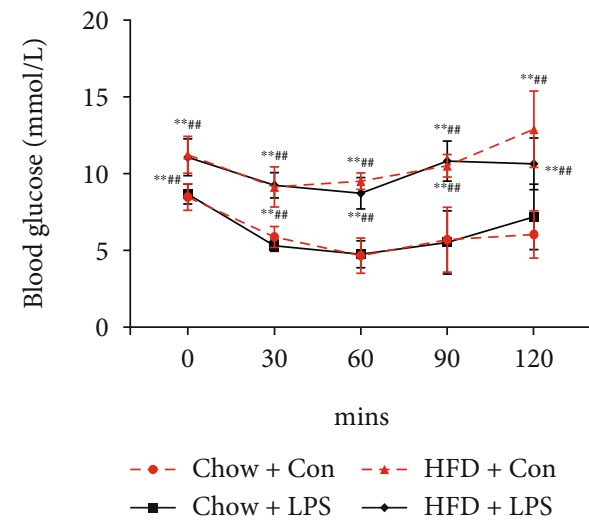

(d)

FIGURE 1: Wild-type mice were challenged with a high-fat diet (HFD) for 12 weeks to induce diabetes. (a) Body weight (BW) gain over time. (b) Food intake was monitored daily for $3 \mathrm{~d}$, and average daily food intake (g) was calculated. (c) Glucose tolerance test (GTT). The total insulin secretion (AUC) is calculated from (c). (d) Insulin tolerance test (ITT) and area under the curve. Each bar represents the mean \pm SEM $(n=6-8)$. Compared with the chow + con group, ${ }^{*} p<0.05$ and ${ }^{* *} p<0.01$. Compared with the chow + LPS group, ${ }^{\#} p<0.05$ and $\# p<0.01$. One-way ANOVA with Tukey's test. Chow+con: mice on 12 weeks of the chow diet received the same volume of solvent without LPS; HDF+con: mice on 12 weeks of the HDF diet received the same volume of solvent without LPS; chow+LPS: mice on 12 weeks of the chow diet subjected to LPS $(10 \mathrm{mg} / \mathrm{kg}$, intraperitoneally) stimulation; HDF+LPS: mice on 12 weeks of the HDF diet subjected to LPS $(10 \mathrm{mg} / \mathrm{kg}$, intraperitoneally) stimulation.

renal microvascular dysfunction, thus exacerbating already severe hypoxia and increasing ROS generation [11]. In diabetic patients, a high blood glucose level increases oxidative stress, leading to increased ROS accumulation and delayed recovery of sepsis-associated AKI [12]. Effective promotion of the clearance or inhibition of ROS production is an important strategy for the treatment of sepsis-associated AKI, particularly in patients with DM.
NADPH oxidases (NOXs) are recognized as the main source of ROS. NOXSs are membrane-spanning enzymes that produce hydrogen peroxide (NOX4, DUOX1-2) or superoxide (NOX1-3, NOX5) in an NADPH-dependent manner [13]. Nox2 and NOX4 are the main NOX subtypes that produce ROS in the kidneys [14-16]. A large accumulation of ROS leads to the formation of cellular ferroptosis. Ferroptosis, a type of programmed cell death, is 


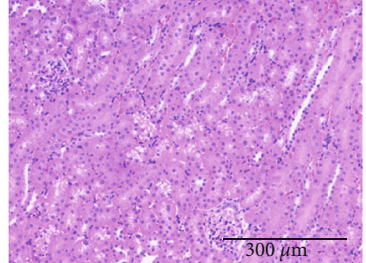

Chow + Con

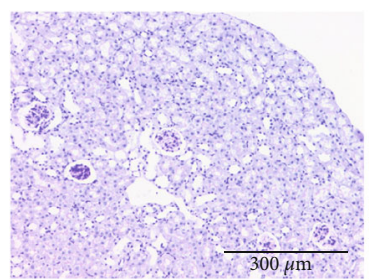

Chow + Con

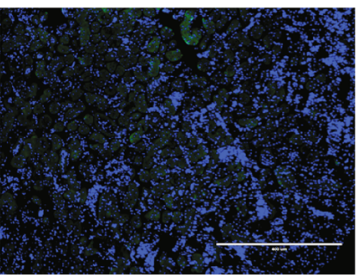

Chow + Con

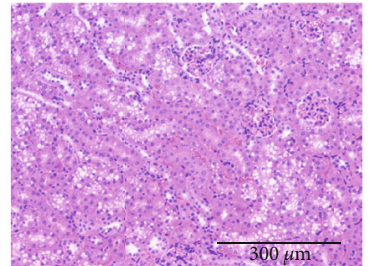

HFD + Con

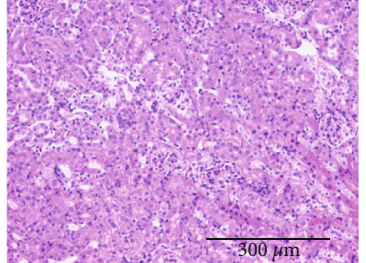

Chow + LPS

(a)

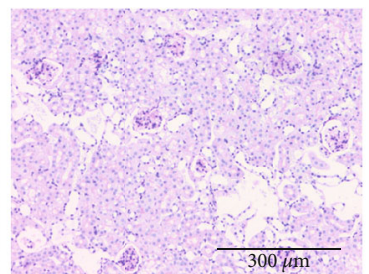

HFD + Con

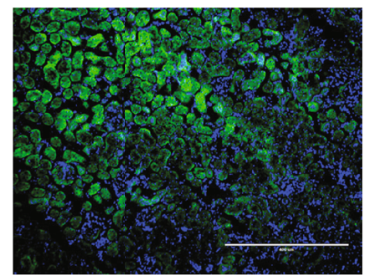

HFD + Con

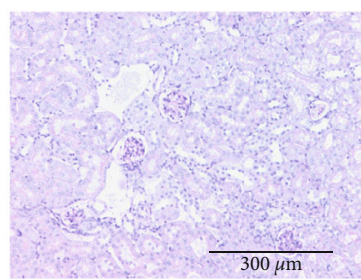

Chow + LPS

(b)

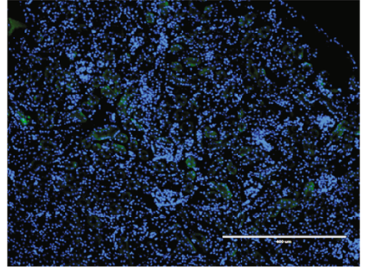

Chow + LPS

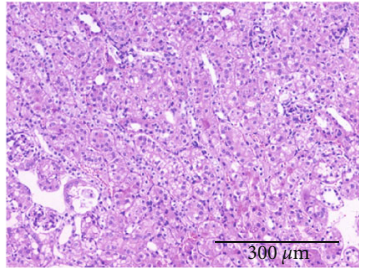

HFD + LPS

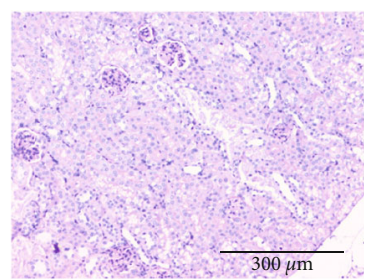

HFD + LPS

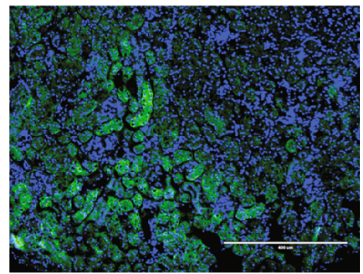

HFD + LPS

(c)

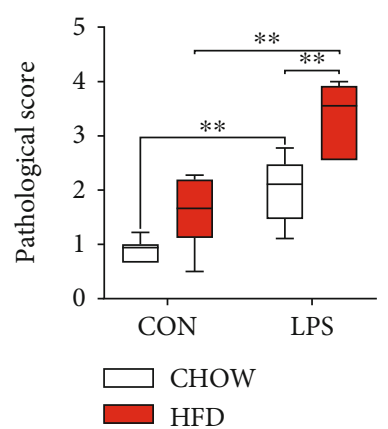

(d)

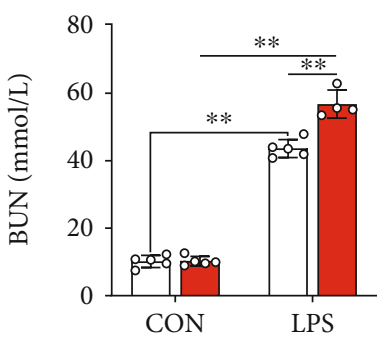

O CHOW

- HFD

(e)

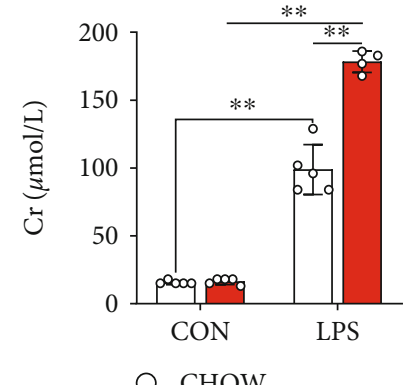

HFD

(f)

FIGURE 2: Continued. 


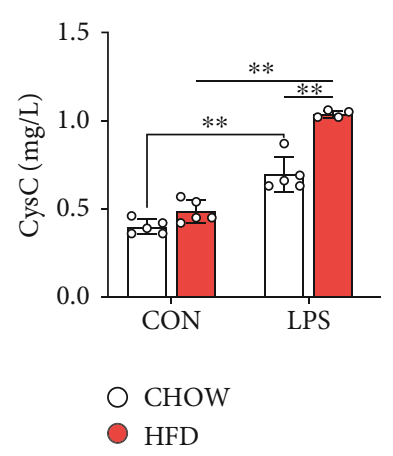

(g)

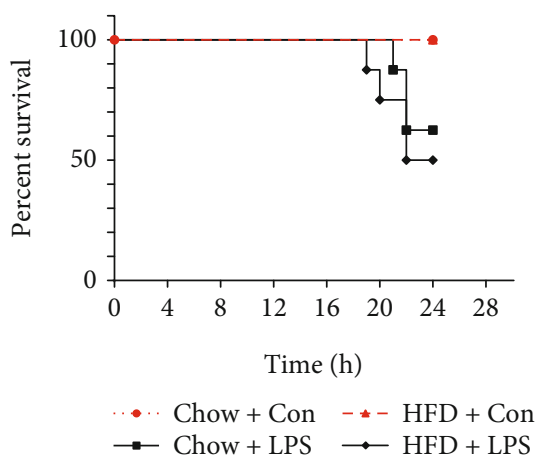

(h)

FIGURE 2: Diabetes-aggravated septic renal injury in mice. Renal pathology was clearly observed via renal H\&E ((a) 200x) and periodic acidSchiff (PAS) staining ((b) 200x). BODIPY staining ((c) 200x) was used to analyze the phospholipid accumulation in the proximal tubular cells of different groups of mice. The pathological score (d) was evaluated according to renal H\&E staining. Serum urea nitrogen (BUN) (e), creatinine (CCr) (f), and cystatin C (Cysc) (g) levels, which reflect renal function, were determined. Furthermore, the survival rate (h) of diabetic mice subjected to LPS was calculated. Each bar represents the mean \pm SEM $(n=4-8)$. ${ }^{*} p<0.05$ and ${ }^{* *} p<0.01$, one-way ANOVA with Tukey's test.

characterized by iron-dependent accumulation of ROS, which can lead to organ dysfunction [17]. Inhibition of the $\mathrm{NADPH}$ oxidase pathway may provide a novel solution to ROS accumulation during sepsis-associated AKI.

The aim of the current study is to investigate the effect of NADPH oxidase inhibition on reduction of renal ROS release and subsequent ferroptosis accumulation and its ability to decrease sepsis mortality by restoring renal function in high-fat diet- (HFD-) challenged mice.

\section{Methods and Materials}

2.1. Animals and Ethics Statement. 5-week-old male C57BL/ 6 mice were purchased from the Model Animal Research Center of Nanjing University and housed in the animal center barrier system $\left(22 \pm 2^{\circ} \mathrm{C}, 50 \pm 5 \%\right.$ humidity, and $12 \mathrm{~h}$ light/dark cycle) and provided with free access to water and food. Obesity was induced by feeding mice a high-fat diet (HFD) containing 60\% fat calories (D12492; Research Diets, USA) for 12 weeks, while mice on a regular rodent chow diet (5\% fat wt./wt.; Laboratory Animal Center of Sun Yat-sen University, China) were used as lean controls. Body weight was evaluated every 2 weeks, and daily food intake was monitored during the dietary intervention. The experimental animal protocol was approved by the Animal Ethics Committee of the Sun Yat-sen University. This committee is standardly guided by the Care and Use of Laboratory Animals (1996).

2.2. Glucose and Insulin Tolerance Tests. Glucose levels were measured at the indicated times with an Optium Xceed glucometer (Abbott Diabetes Care, Inc., Alameda, CA). For the oral gavage glucose tolerance test (OGTT), mice were fasted for $16 \mathrm{~h}$ overnight, then treated with intragastric glucose ( $2 \mathrm{~g} / \mathrm{kg}$ wt.), and tail vein blood glucose levels were measured at $0,30,60,120$, and $180 \mathrm{~min}$. For the intraperitoneal insulin tolerance test (ipITT), mice were fasted for $6 \mathrm{~h}$ and then injected intraperitoneally with insulin $(0.65 \mathrm{U} / \mathrm{kg} \mathrm{wt}$.$) , and$ tail vein blood glucose levels were measured at $0,30,60$, and $120 \mathrm{~min}$.

2.3. LPS-Induced Septic Kidney Injury and Treatment. After dietary intervention, mice were subjected to various treatments. The mice were treated with a single dose of LPS $(10 \mathrm{mg} / \mathrm{kg}$, L2880, Sigma-Aldrich, Germany) or phosphatebuffered saline (PBS) via intraperitoneal injection.

For ferroptosis inhibition experiments, mice were injected intraperitoneally with ferrostatin-1 $(10 \mathrm{mg} / \mathrm{kg}$, HY100579, MedChemExpress, USA) or vehicle $1 \mathrm{~h}$ before LPS administration. For NADPH oxidase inhibition, Vas 2870 $(10 \mathrm{mg} / \mathrm{kg}, \mathrm{HY}-12804$, MedChemExpress, USA) or vehicle was injected intraperitoneally $3 \mathrm{~h}$ before LPS administration. Twenty-four hours after LPS or PBS injection, mice were euthanized, and blood and kidneys were collected for further analysis.

\subsection{Hematoxylin-Eosin Staining and Periodic Acid-Schiff} Staining. Fresh kidney tissues were collected, fixed in $4 \%$ neutral formalin buffer solution (HT50-1-2; Sigma-Aldrich, Germany), and sectioned (5 $\mu \mathrm{m})$. Hematoxylin-eosin (HE) staining was used for histopathological analysis using a light microscope (Leica Corporation, Germany). Tubular injuries were graded by a score of $0-4$ ( 0 , no change; 1 , change affecting $<25 \%$ of the field; 2 , change affecting $25 \%-50 \%$ of the field; 3 , change affecting $50 \%-75 \%$ of the field; and 4 , change affecting $>75 \%$ of the field). Glycogen storage in kidney tissue was analyzed with a periodic acid-Schiff staining kit (G1008, Servicebio, China) following the manufacturer's instructions. Other fresh tissues were put into liquid nitrogen and sliced. The fat content of renal tubular epithelial cells was analyzed using the BODIPY staining kit (D3922, Thermo, USA) according to the manufacturer's instructions.

2.5. Serum Urea Nitrogen (BUN), Creatinine (CCr), Cystatin $C$ (Cysc), and Glutathione Reductase (GR) Measurement. BUN, CCr, Cysc, and GR levels were analyzed by the 


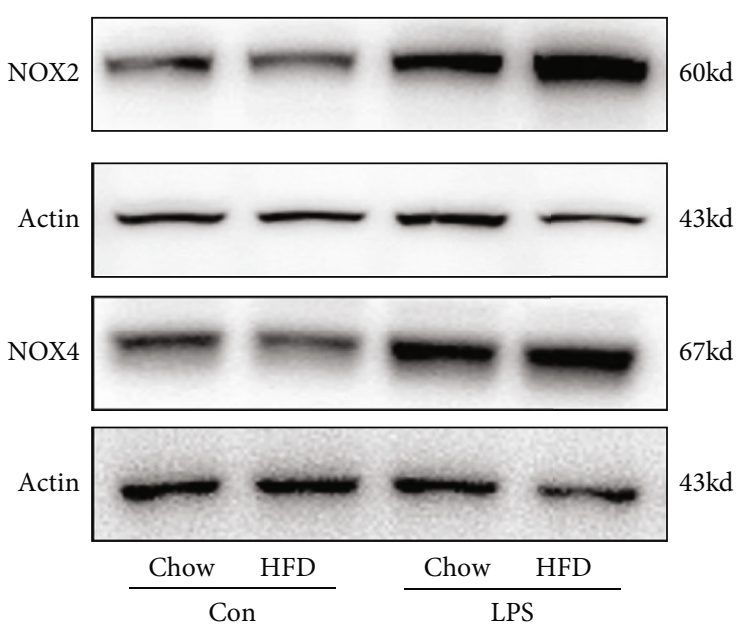

(a)

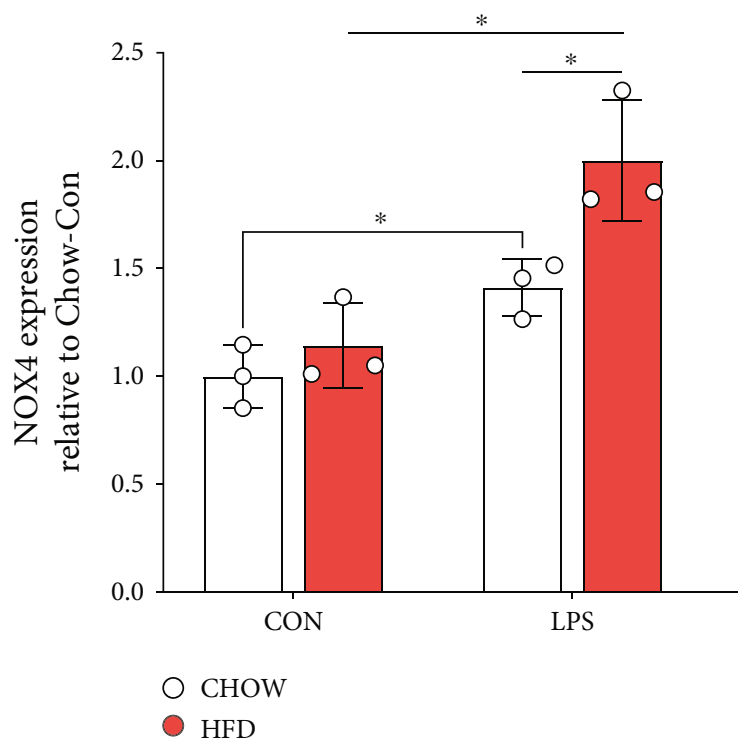

(c)

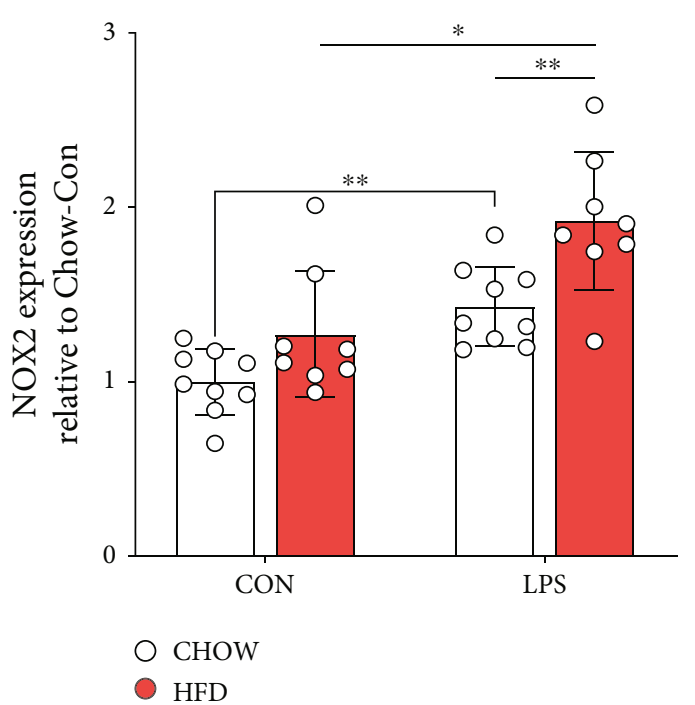

(b)

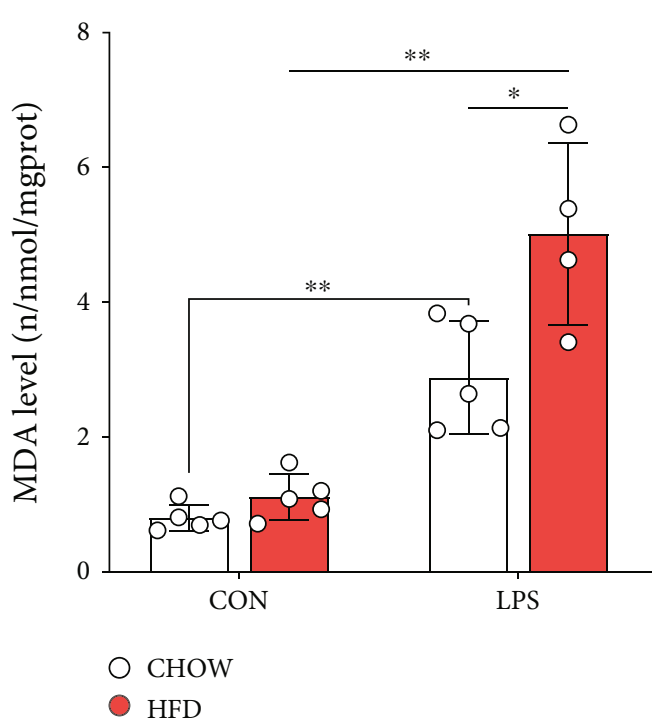

(d)

FIGURE 3: Renal NADPH oxidase subunits were activated in diabetic mice subjected to LPS. Renal NADPH subunits, NOX2 and NOX4, were detected by western blotting (a). Gray analysis was performed according to the bands of NOX2 (b) and NOX4 (c). Renal malondialdehyde (MDA) (d) expression was measured using enzyme-linked immunosorbent assay (ELISA). Each bar represents the mean $\pm \operatorname{SEM}(n=3-8) .{ }^{*} p<0.05$ and ${ }^{* *} p<0.01$, one-way ANOVA with Tukey's test.

automatic biochemistry analyzer (Watford Olympus AU640, United Kingdom).

2.6. ROS Measurement. The ROS levels in the kidney were detected using a DCFH-DA assay kit (BB-470536, Best Bio, China). After washing with cleaning solution at room temperature, the tissues were incubated with the DCFH-DA staining [18] solution for $1 \mathrm{~h}$ at $37^{\circ} \mathrm{C}$, and images were captured using a fluorescence microscope (EVOS FL, Life Technology, USA).

2.7. Malondialdehyde (MDA) Level Measurement. MDA levels were measured using an MDA-specific assay reagent kit (A003-1-1, Jiancheng, China) according to the manufac- turer's instructions, and the absorbance was measured at $532 \mathrm{~nm}$ using a microplate reader (ELX800, Bio-Tek, USA). The protein concentration of each sample was measured using a BCA Protein Assay kit (BCA-23225, Thermo Fisher Scientific, USA).

2.8. Transmission Electron Microscopy (TEM). Kidney tissues were fixed with $4 \%$ glutaraldehyde solution (G1102, Servicebio, China) for $12 \mathrm{~h}$, stained with $1 \%$ osmium tetroxide (18459, Ted Pella Inc., USA), dehydrated with acetone, and embedded in resin. Samples were then sectioned $(60 \mathrm{~nm})$, mounted on copper grids, stained, and examined using TEM (HT7700, Hitachi, Japan). 

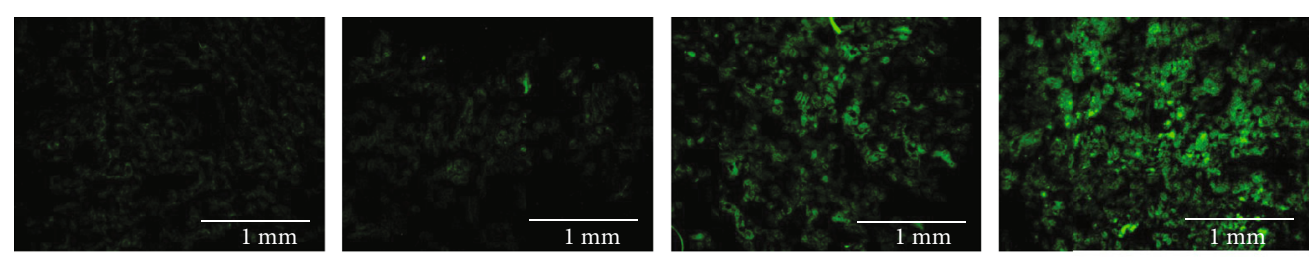

ROS
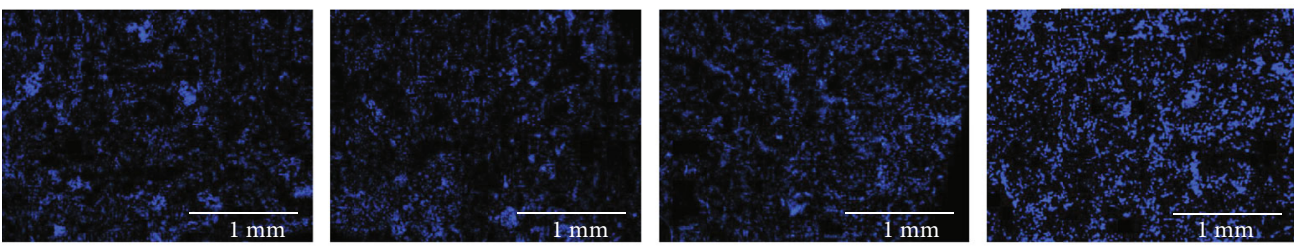

DAPI
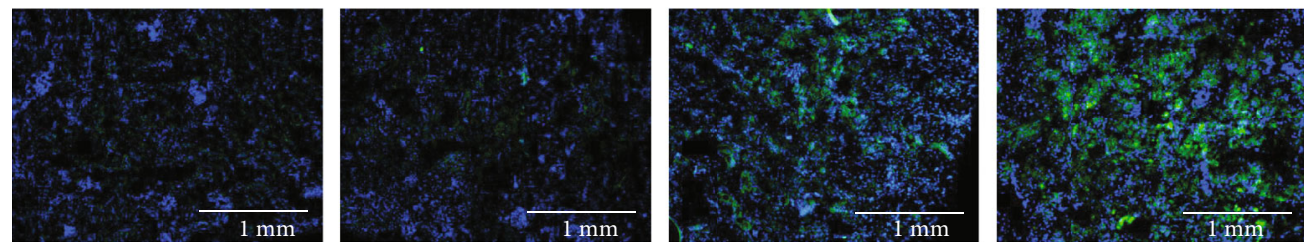

Chow + LPS

HFD + LPS

(a)

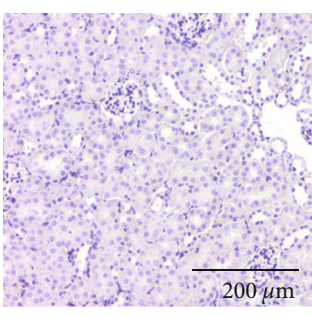

Chow + Con
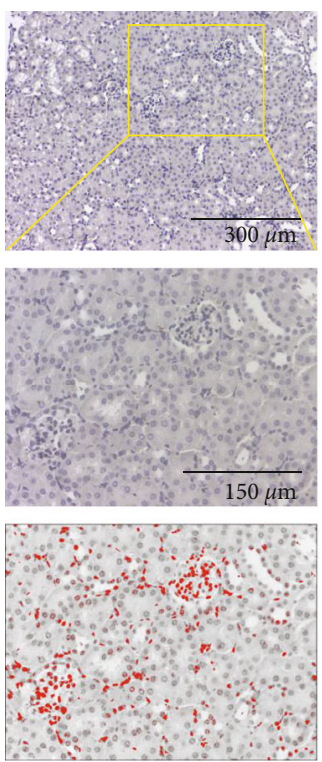

Chow + Con

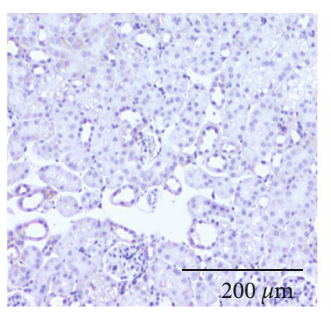

HFD + Con
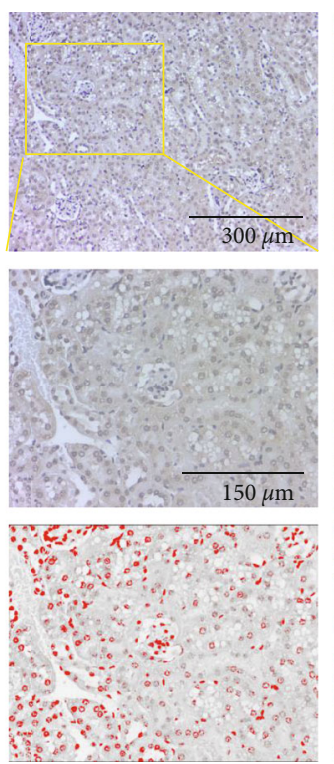

HFD + Con

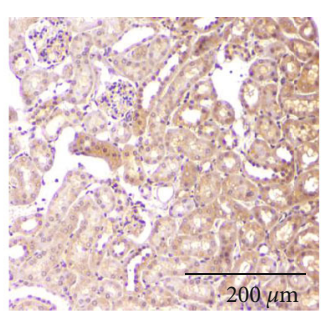

Chow + LPS

(b)
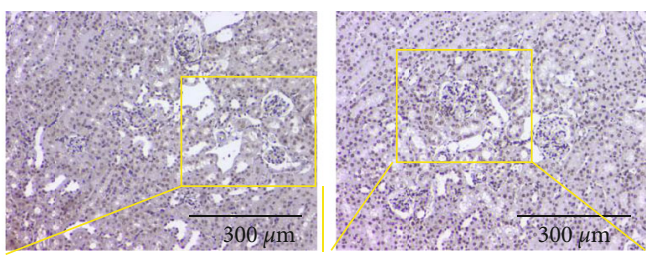

$200 x$
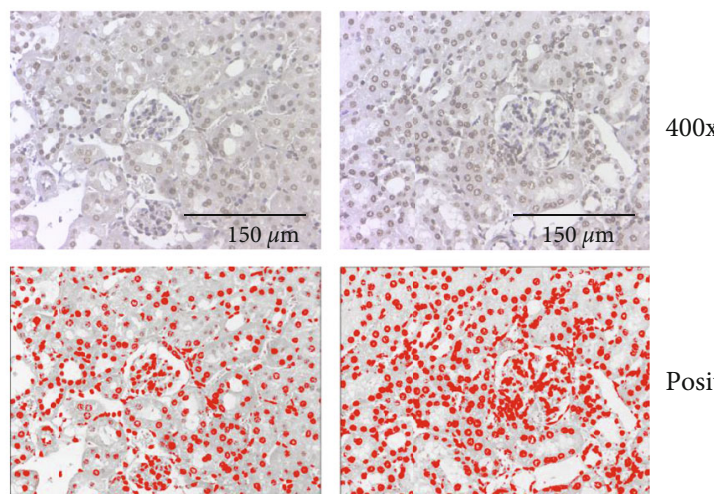

Chow + LPS
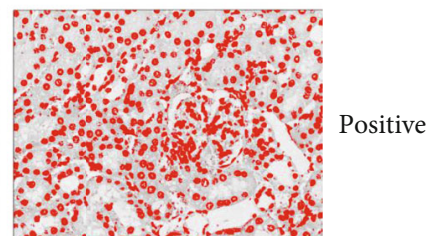

HFD + LPS

(c)

FIgUre 4: Continued. 


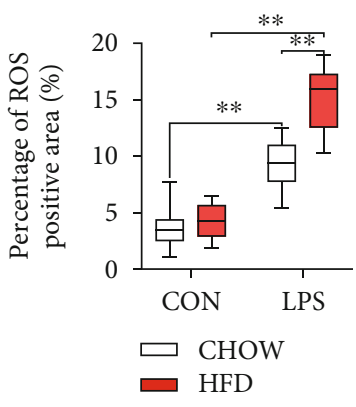

(d)

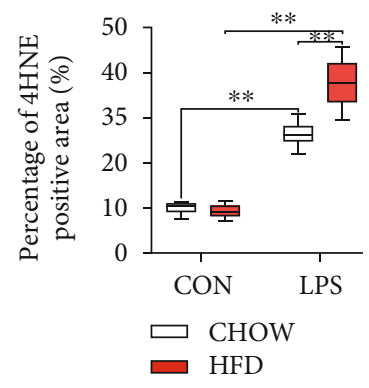

(e)

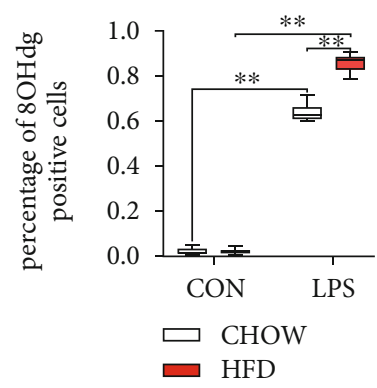

(f)

FIGURE 4: Diabetes-exacerbated oxidative stress during septic renal injury. Renal reactive oxygen species (ROS) levels were detected by immunofluorescence (a). Renal 4-hydroxynonenal (4-HNE) immunohistochemical staining (b) and 8-hydroxydeoxyguanosine (8-OHdG) immunohistochemical staining (c) were performed to evaluate oxidative stress levels in the kidneys. Semiquantitative analysis of ROS immunofluorescence staining (d), 4-HNE immunohistochemical staining (e), and 8-OHdG immunohistochemical staining (f). Each bar represents the mean \pm SEM $(n=6-8) .{ }^{*} p<0.05$ and ${ }^{* *} p<0.01$, one-way ANOVA with Tukey's test.

2.9. Immunohistochemistry Staining. After dewaxing and dehydration, thin sections $(5 \mu \mathrm{m})$ of kidney tissues were deparaffinized, blocked, and incubated with anti-4-HNE (1:200; ab46545, Abcam Technology, United Kingdom), anti-8-OHDG (1:800; ab48508, Abcam Technology, United Kingdom), and anti-GPX4 (1:200; ab125066, Abcam Technology, United Kingdom) $12 \mathrm{~h}$ at $4^{\circ} \mathrm{C}$. Sections were stained with an HRP IHC kit (GK500710, Gene Tech, China) according to the manufacturer's instructions. Staining intensity was observed under a light microscope (Leica Corporation, Germany) and analyzed using ImageJ software (National Institutes of Health, USA). Finally, ImageJ (V1.8.0.112, National Institutes of Health, USA) was used to analyze the positive area in each slide and make statistics.

2.10. Western Blotting. Mouse kidneys were homogenized and centrifuged at $13000 \mathrm{rpm}$ at $4^{\circ} \mathrm{C}$ for 30 minutes to take the supernatant. Extracts of the kidney tissue were prepared in lysis buffer. The total protein was separated on $5-20 \%$ acrylamide gel by using twelve alkyl sulfate polyacrylamide gel electrophoresis (SDS-PAGE) and then transferred to polyvinyl fluoride two (PVDF). After sealing with 5\% fatfree milk for 1 hour, the membrane was incubated with the corresponding primary anti-rabbit antibody at $4^{\circ} \mathrm{C}$ overnight. Whole-cell lysates of the kidney were analyzed using western blotting to detect the expression of NOX2, NOX4, ACSL4, GPX4, FTH1, and $\beta$-actin. Primary antibodies against Gp91phox/NOX2 (1 : 1000; ab129068, Abcam Technology, United Kingdom), NOX4 (1:1000; ab133303, Abcam Technology, United Kingdom), ACSL4 (1:1000; A6826, ABclonal Technology, China), GPX4 (1:1500; ab125066, Abcam Technology, United Kingdom), FTH1 (1:1000; ab75972, Abcam Technology, United Kingdom), and $\beta$-actin ( 1 : 10000; AC026, ABclonal Technology, China) were used. The imprinting was visualized by enhanced chemiluminescence (ECL) system, and the gray value was scanned and quantified by ImageJ software.

2.11. Statistical Analysis. All data were analyzed using SPSS version 22.0. Quantitative data are presented as the mean \pm SEM from multiple samples ( $n=6-10$ for each group). The Kolmogorov-Smirnov test was used to test the normality of the data, and Levene's test was used to test the homogeneity of variance. The $t$-test was used to analyze the differences between two groups, followed by the LSD post hoc test. Comparisons of multiple groups were analyzed using two-way repeated measures analysis of variance (ANOVA) or one-way ANOVA. Differences were considered statistically significant when $p$ values were less than 0.05 .

\section{Results}

3.1. High-Fat Diet-Induced Diabetes Aggravated Septic Renal Injury. From the second week of HFD intervention, a significant increase in body weight was observed in the HFD group $(p<0.01)$, which persisted until the end of intervention (Figure 1(a)). No differences in food intake were observed between the HFD and chow-diet groups (Figure 1(b)). The GTT results showed deteriorated glucose intolerance in HFD-challenged mice with a significantly larger AUC value (Figure 1(c)). Additionally, higher blood glucose levels at time points $0,30,60,90$, and $120 \mathrm{~min}$ with a higher AUC value were observed in HFD-challenged mice during the ITT (Figure 1(d)).

The results of H\&E (Figures 2(a) and 2(b)) showed that high-fat diet-induced diabetes aggravated septic renal injury and caused fat accumulation in the proximal tubular cells (Figure 2(c)), which had higher injury scores (Figure 2(d)) in HFD-challenged mice than those in chow diet-fed mice when subjected to LPS stimulation. Moreover, the serum BUN (Figure 2(e)), CCr (Figure 2(f)), and Cysc (Figure 2(g)) levels, which reflect renal function, were significantly increased in HFD-challenged mice compared with those in chow diet-fed mice when subjected to LPS stimulation. LPS stimulation led to higher motility in HFDchallenged mice than in chow-fed mice. As shown in Figure 2(h), the LPS stimulation significantly decreased the survival rate of mice which was lower in HFD-challenged mice than those in chow diet-fed mice. 

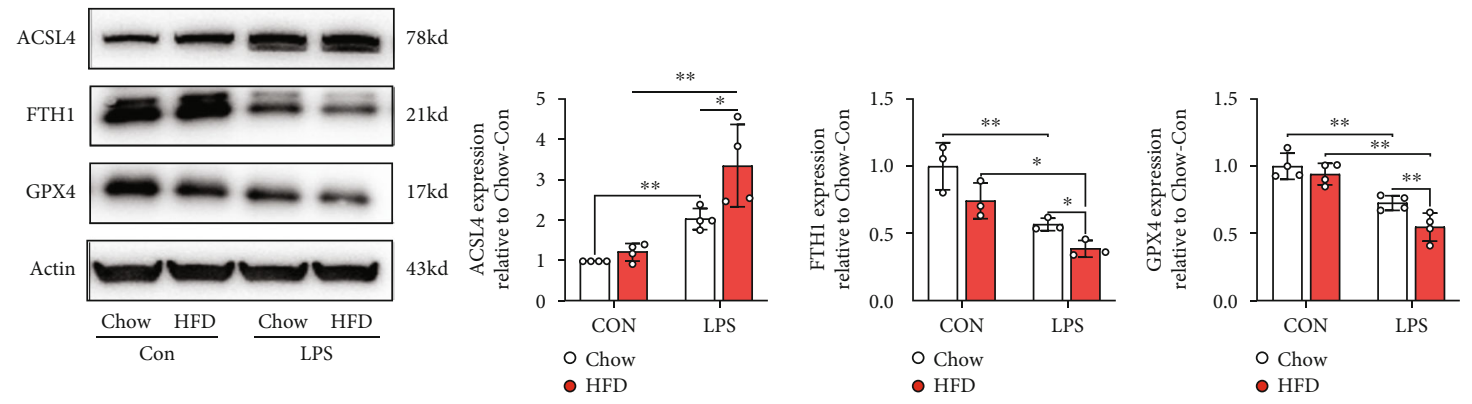

(a)
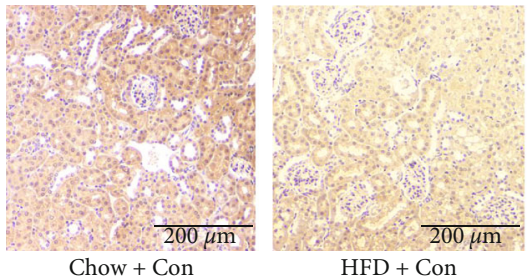

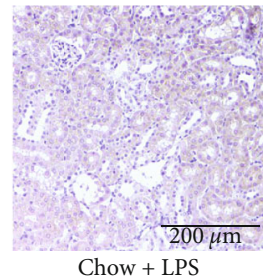

(b)
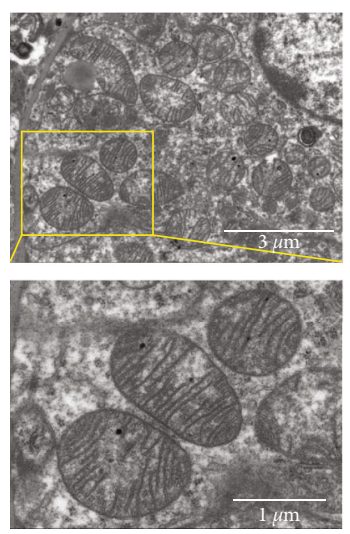

Chow + Con
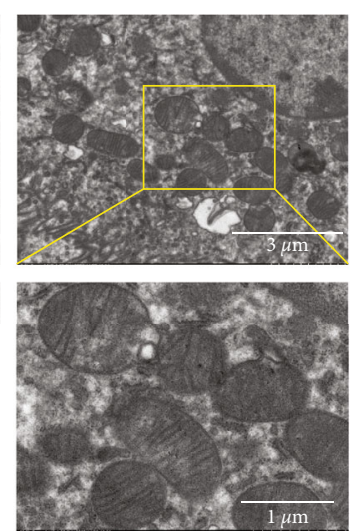

HFD + Con

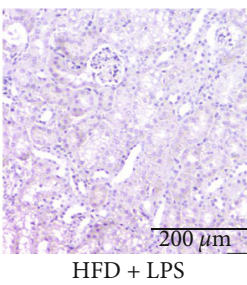

HFD + LPS

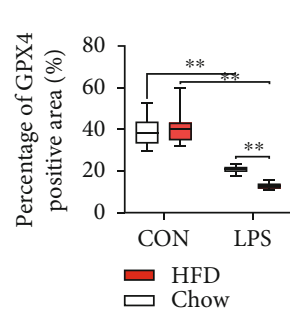

$\square$ HFD

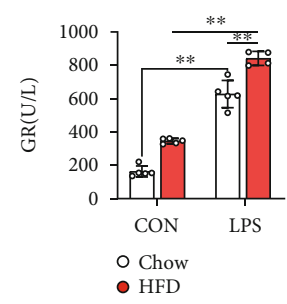

(c)
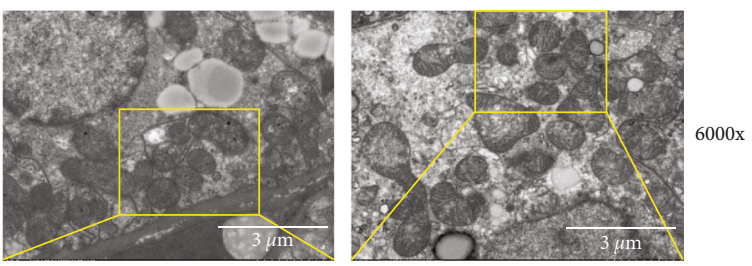

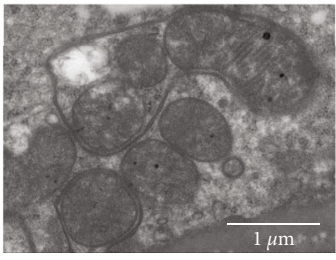

Chow + LPS

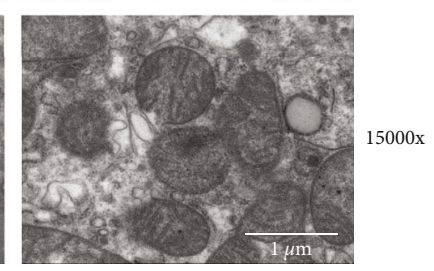

HFD + LPS

(d)

Figure 5: Renal ferroptosis accumulated during septic renal injury in diabetic mice. The expression levels of ferroptosis-related proteins including ASCL4, FTH1, and GPX4 were determined by western blotting (a). GPX4 levels were also detected by immunohistochemical staining (b). The renal scavenging effect on ROS is reflected by the glutathione reductase (GR) level (c). Morphological features of mitochondria were measured using electron microscopy (d). Each bar represents the mean \pm SEM $(n=3-6)$. ${ }^{*} p<0.05$ and ${ }^{* *} p<0.01$, one-way ANOVA with Tukey's test.

\subsection{NADPH Oxidase and Ferroptosis Were Activated during} Septic Renal Injury in HFD-Challenged Diabetic Mice. To investigate the underlying mechanism of HFD-challenged diabetes-deteriorated septic renal injury, NADPH oxidase activation and changes in ferroptosis accumulation were measured. As shown in Figures 3(a)-3(c), levels of NADPH oxidase subunits, NOX2 and NOX4, significantly increased in HFD-challenged mice compared to those in chow-fed mice when subjected to LPS stimulation. Additionally, LPS stimulation led to higher renal MDA (Figure 3(d)), ROS (Figure 4(a), Figure 4(d)), 4-HNE (Figure 4(b), Figure 4(e)), and 8-OHdG expression levels (Figures 4(c) and $4(\mathrm{f})$ ) in chow-fed mice. HFD-induced diabetes can aggravate renal oxidative stress via NADPH oxidase subunit activation.
As ROS accumulation leads to ferroptosis, we detected ferroptosis in renal sepsis. As shown in Figures 5(a) and 5(b), ferroptosis-related protein ASCL4 significantly increased in LPS-stimulated groups, and ASCL4 expression was higher in HFD-challenged mice than that in chow-fed mice when subjected to LPS stimulation. Additionally, a decrease in the levels of ferroptosis-related proteins FTH1 and GPX4 was observed in HFD-challenged mice subjected to LPS stimulation $(p<0.01)$. Simultaneously, glutathione reductase (GR) level (Figure 5(c)), which is involved in the formation of ferroptosis by regulating glutathione (GSH) generation through the NADPH pathway, was significantly increased in HFDchallenged mice than in chow-fed mice when subjected to LPS stimulation. To verify this observation, ferroptosis in renal tubular epithelial cell mitochondria was detected by 

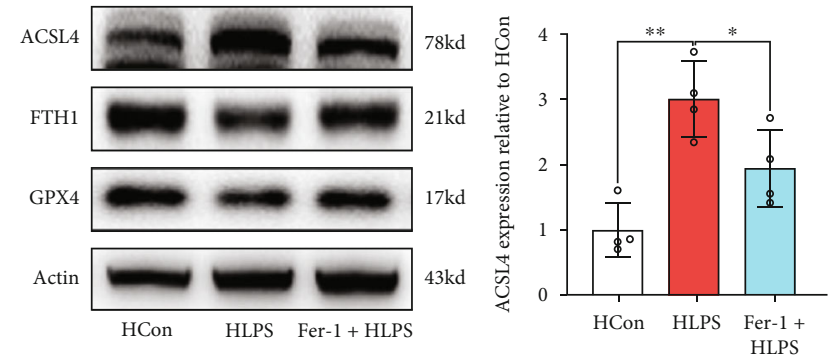

(a)
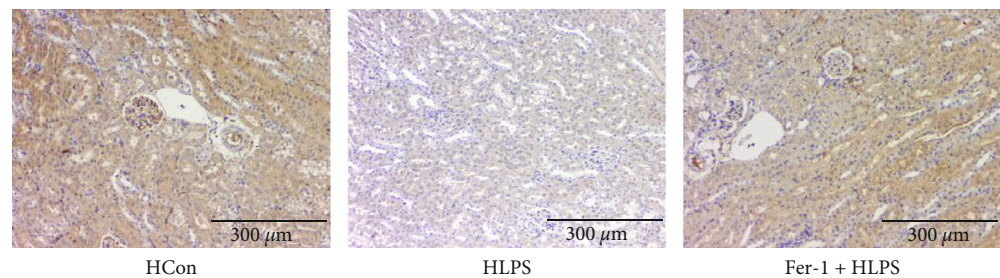

(b)
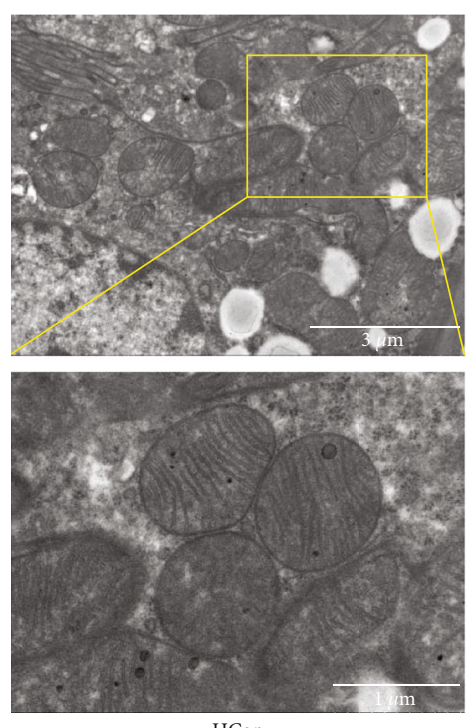

HCon
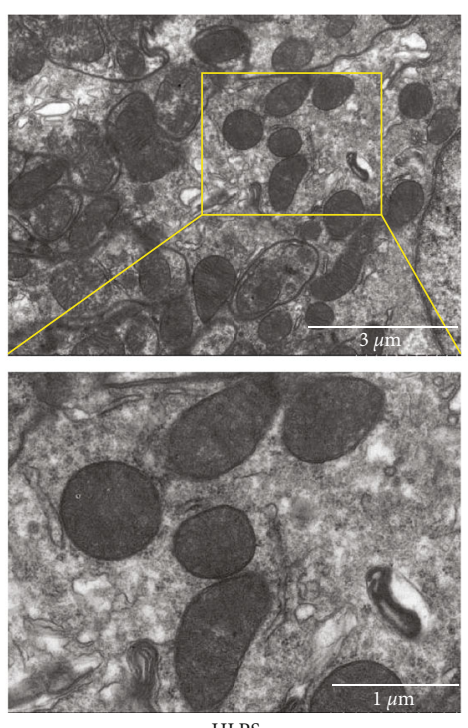

HLPS

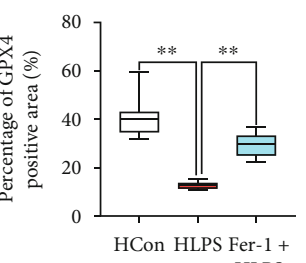

HLPS
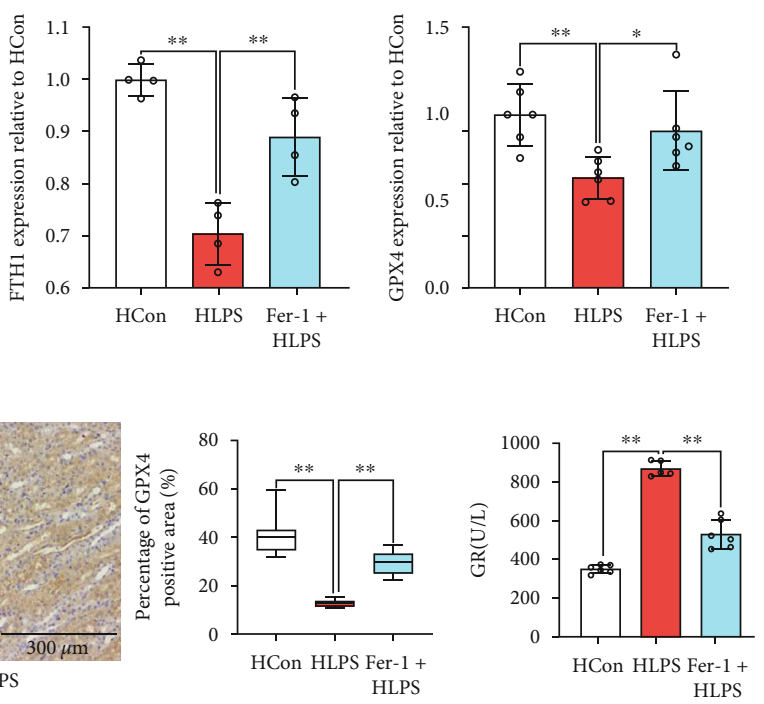

(c)

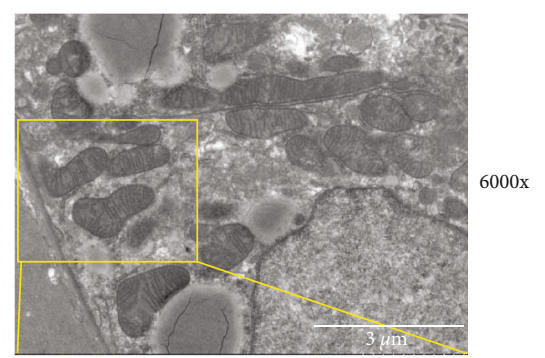

(d)

Figure 6: Fer-1 effectively inhibited ferroptosis accumulation during septic renal injury in diabetic mice. The expression levels of ferroptosis-related proteins, including ASCL4, FTH1, and GPX4, were determined by western blotting (a). GPX4 levels were also detected by immunohistochemical staining (b). The GR level is indicative of the renal scavenging effect on ROS (c). Morphological features of mitochondria were measured using electron microscopy (d). Each bar represents the mean \pm SEM $(n=3-8)$. ${ }^{*} p<0.05$ and ${ }^{* *} p<0.01$, one-way ANOVA with Tukey's test. Hcon: mice fed a HDF diet for 12 weeks received the same volume of solvent without LPS; HLPS: mice fed a HDF diet for 12 weeks and subjected to LPS $(10 \mathrm{mg} / \mathrm{kg}$, intraperitoneally) stimulation; Fer-1+HLPS: ferrostatin-1 (Fer-1) $(10 \mathrm{mg} / \mathrm{kg})$ was administered intraperitoneally $1 \mathrm{~h}$ before LPS administration.

electron microscopy (Figure 5(d)). We found that the cell membrane was broken and vacuolated, mitochondrial spine was decreased or absent, and membrane density was increased under LPS stimulation, which was deteriorated in HFD-challenged mice.

3.3. Ferroptosis Inhibitor Reversed Aggravated Septic Renal Injury in HFD-Challenged Mice. To investigate the role of ferroptosis in septic renal injury in HFD-challenged mice, the ferroptosis inhibitor ferrostatin-1 (Fer-1) was used in vivo. As shown in Figures 6(a) and 6(b), ferroptosis accumulation induced by LPS stimulation in HFD-challenged mice was inhibited by Fer-1, as evidenced by an increase in ASCL4 protein expression and a decrease in FTH1 and GPX4 proteins. However, we found that there were no significant differences in NOX protein expressions between the Fer-1-treated groups and LPS-treated HFD diabetic mice (Supplemental Figure 1). Additionally, the expression of GR was significantly decreased by Fer-1 in the HLPS group compared to that in the HFD-challenged mice under LPS 


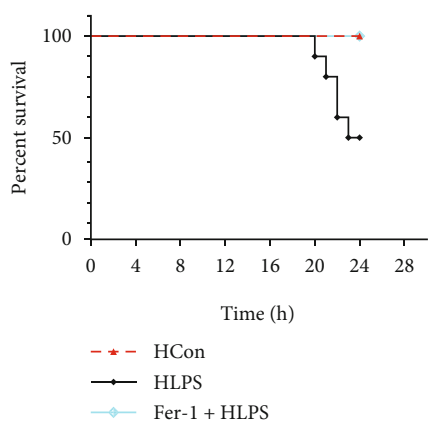

(a)

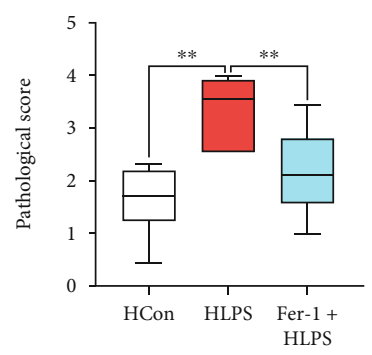

(c)
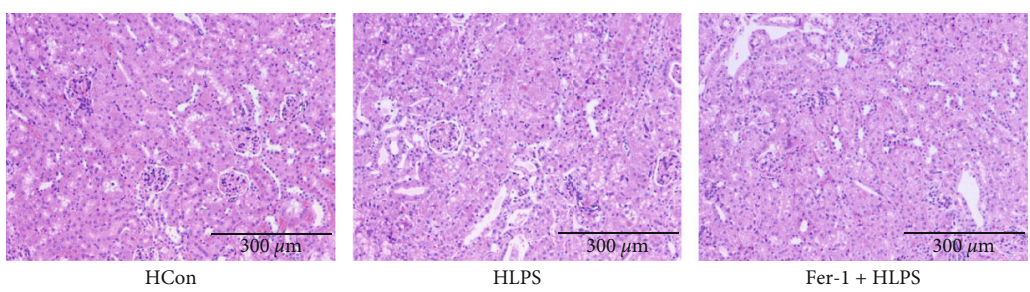

(b)

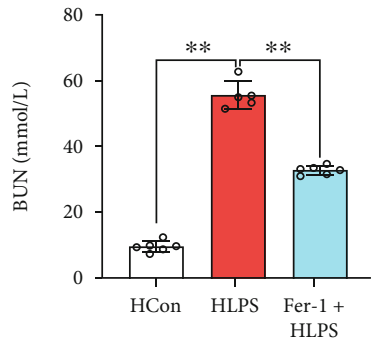

(d)

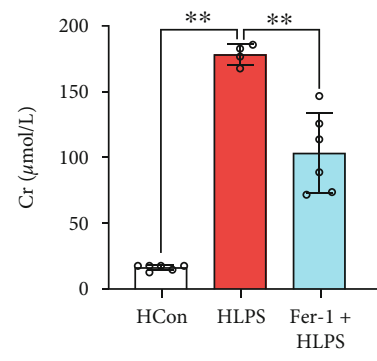

(e)

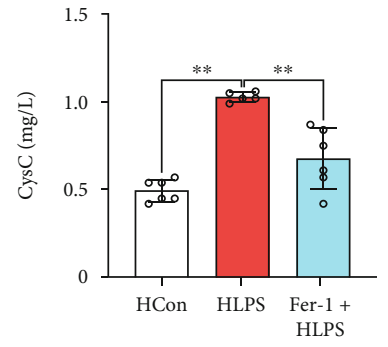

(f)

FIgURE 7: Inhibition of ferroptosis reduced septic renal injury. The survival rate (a) of the mice was calculated. Renal pathology was observed via renal H\&E staining ((b) 200x). The pathological score (c) was evaluated according to renal H\&E staining. Serum BUN (d), CCr (e), and Cysc (f) levels were determined. Each bar represents the mean \pm SEM $(n=6-10)$. ${ }^{*} p<0.05$ and ${ }^{* *} p<0.01$, one-way ANOVA with Tukey's test.

stimulation (Figure 6(c)). Moreover, the mitochondrial changes induced by LPS stimulation in HFD-challenged mice were restored after Fer-1 treatment (Figure 6(d)).

Thereafter, we investigated the effects of Fer-1 on renal pathology and function. As shown in Figure 7(a), Fer-1 treatment effectively increased the survival rate of HFDchallenged mice subjected to LPS stimulation. Moreover, the pathology (Figure 7(b)) and renal function, which were impaired during LPS stimulation, were restored after Fer-1 treatment in HFD-challenged mice, as evidenced by decreased pathological scores (Figure 7(c)) and expression levels of serum BUN (Figure 7(d)), CCr (Figure 7(e)), and Cysc (Figure 7(f)).

3.4. NADPH Inhibition Ameliorated Septic Renal Injury via Suppressing Ferroptosis Accumulation in HFD-Challenged Mice. As ROS accumulation leads to ferroptosis and NADPH oxidase plays an important role in ROS generation, we used Vas2870, to investigate the restoration of renal function by inhibition of NADPH oxidase, which suppresses ferroptosis accumulation. As shown in Figure 8(a), inhibition of NADPH oxidase by Vas2870 increased the survival rate of HFD-challenged mice subjected to LPS stimulation. Moreover, the pathology (Figures 8(b) and 8(c)) and renal function, which were impaired during LPS stimulation, were restored after Vas2870 treatment in HFD-challenged mice, as evidenced by lower levels of serum BUN (Figure 8(d)), CCr (Figure 8(e)), and Cysc (Figure 8(f)).

Compared to HFD-challenged mice treated with solvent, NADPH oxidase inhibition resulted in a significant decrease in renal oxidative stress, as evidenced by lower MDA $(p<0.05)$ (Figure $8(\mathrm{~g})), 4$-HNE $(p<0.05)$ (Figure $8(\mathrm{~h})$, Figure $8(\mathrm{k})$ ), ROS generation $(p<0.05)$ (Figure $8(\mathrm{i})$, Figure $8(\mathrm{l}))$, and 8 -OHdG $(p<0.05)$ (Figures $8(\mathrm{j})$ and $8(\mathrm{~m})$ ) expression levels in HFD-challenged mice subjected to LPS stimulation. The expressions of NADPH oxidase subunits, NOX2 and NOX4, which were activated by LPS stimulation, were significantly decreased after Vas2870 treatment in HFD-challenged mice (Figure 8(n)).

Furthermore, the results of ferroptosis accumulation (Figures 9(a) and 9(b)) showed that ASCL4 protein expression increased, whereas FTH1 and GPX4 protein expression decreased after Vas2870 treatment in HFD-challenged mice subjected to LPS stimulation. This was accompanied by a significant decrease in GR levels after Vas2870 application in the HLPS group compared to that in the HFD-challenged mice under LPS stimulation $(p<0.05)$ (Figure 9(c)). Additionally, mitochondrial changes induced by LPS stimulation were restored by Vas2870 in HFD-challenged mice (Figure 9(d)). These results indicated that NADPH inhibition could restore septic renal function from injury by suppressing ferroptosis accumulation in HFD-challenged mice.

\section{Discussion}

The effect of obesity or type 2 diabetes on the survival of sepsis-associated AKI remains a controversial topic [19, 20]. In the current study, mice subjected to 12 weeks of a HDF diet were successfully obtained to mimic type 2 diabetes. We found that HFD-induced diabetes aggravated renal 


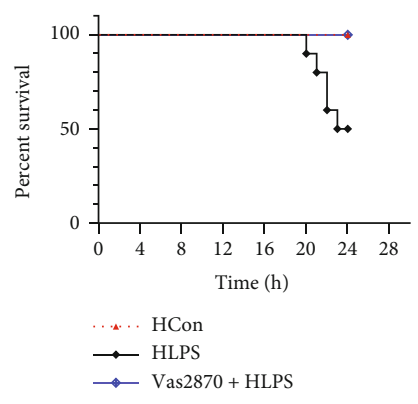

(a)

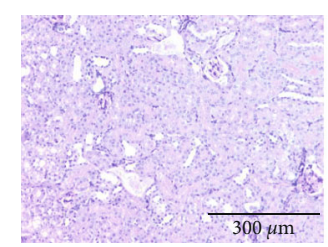

HCon

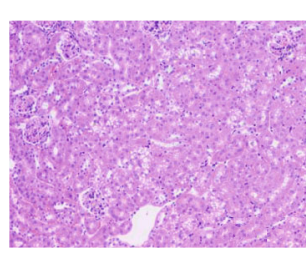

HCon

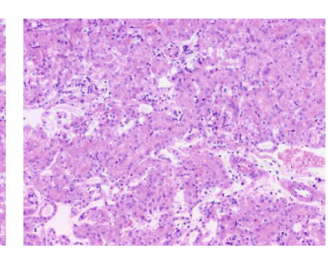

HLPS

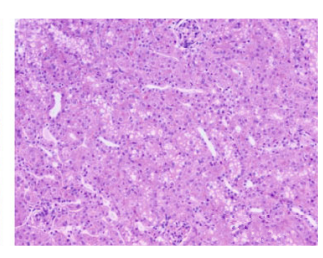

Vas $2870+$ HLPS

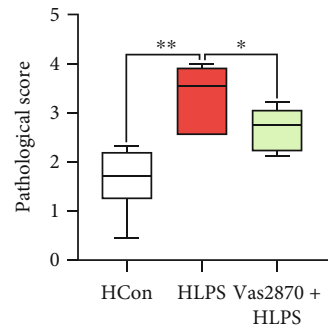

(b)

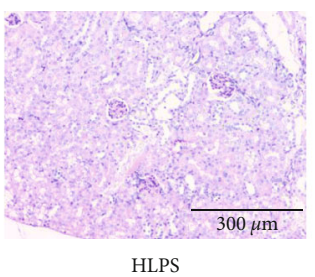

HLPS

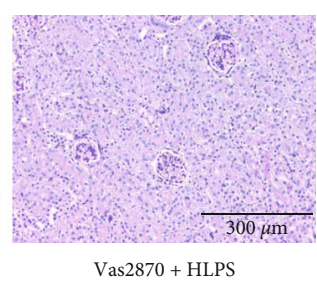

Vas $2870+$ HLPS

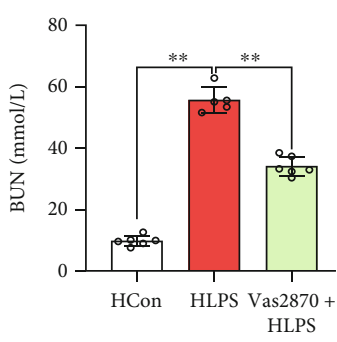

(d)

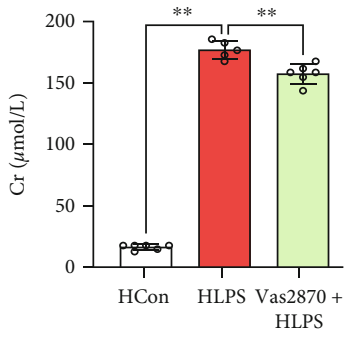

(e)

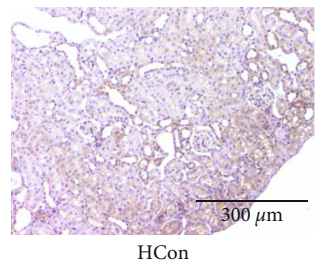

(c)

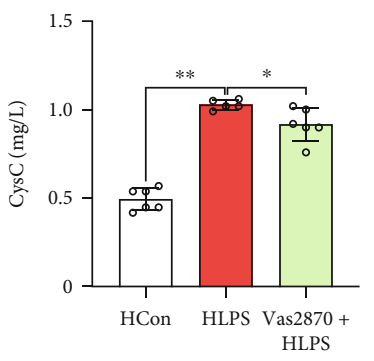

(f)

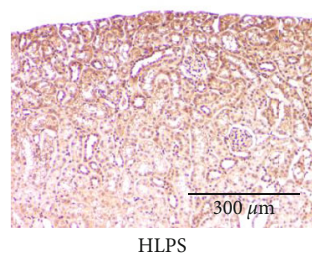

(h)

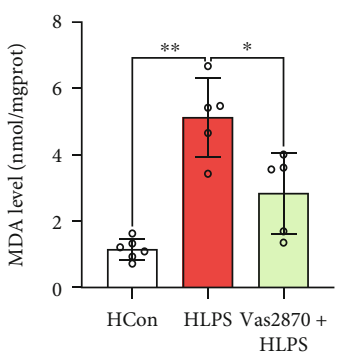

(g)

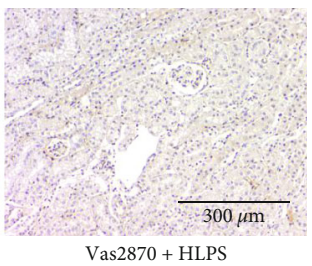

Figure 8: Continued. 

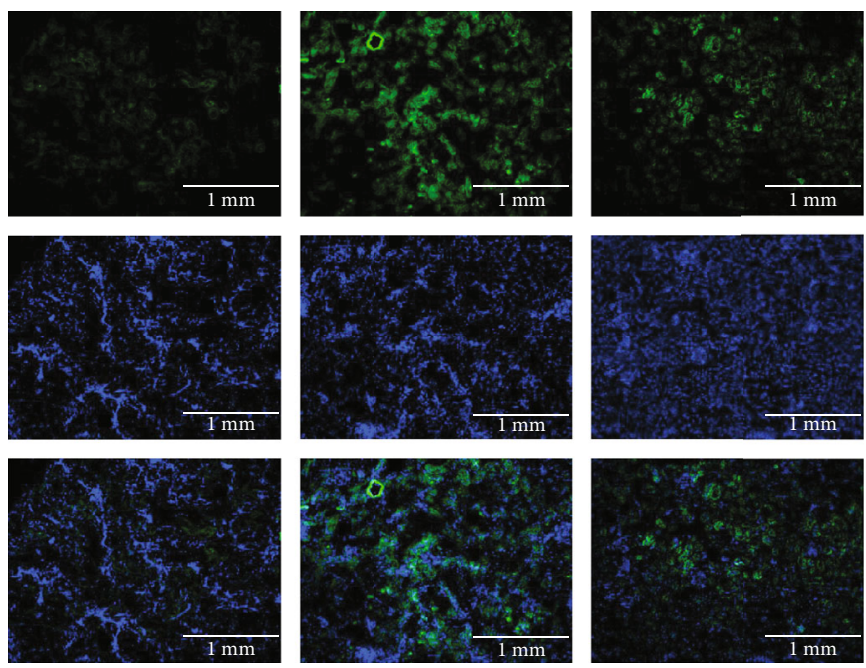

HCon

HLPS

Vas2870 + HLPS

(i)
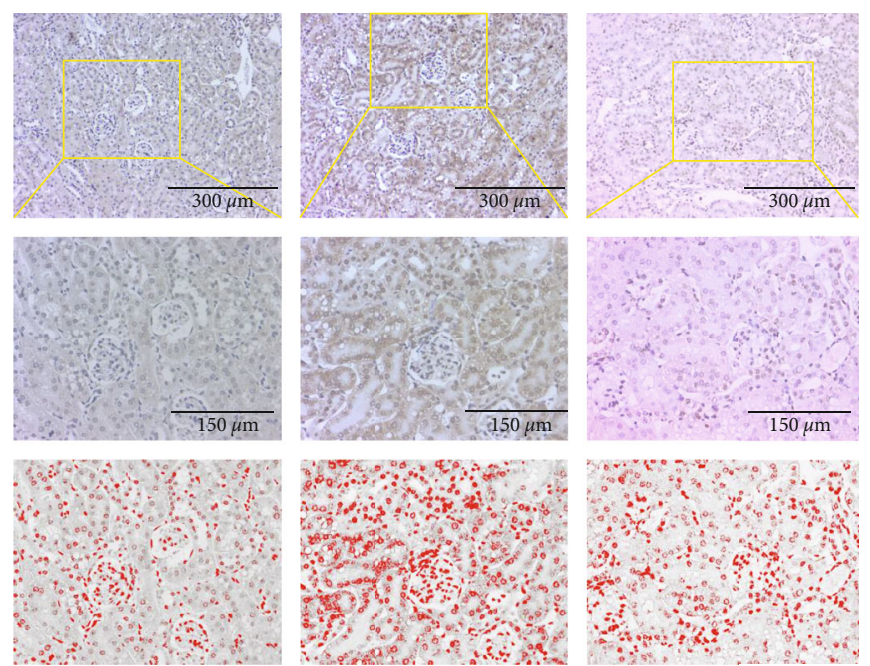

HCon

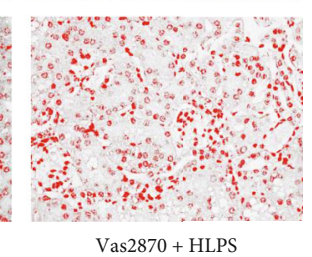

(j)

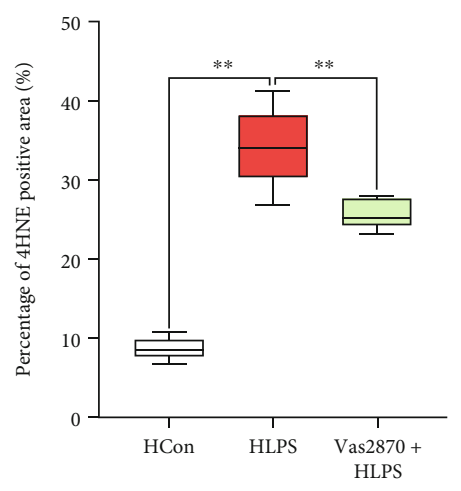

(k)

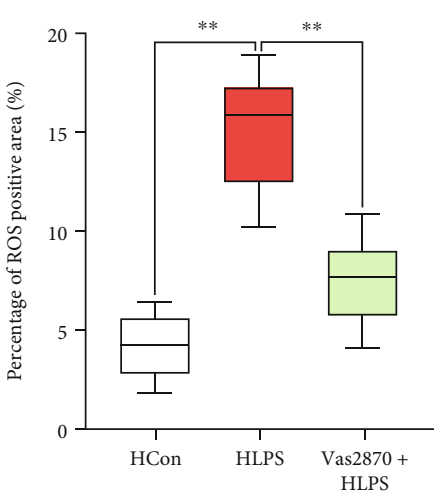

(l)

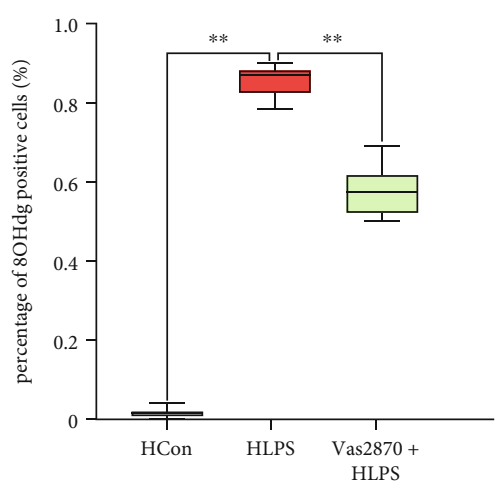

(m)

Figure 8: Continued. 

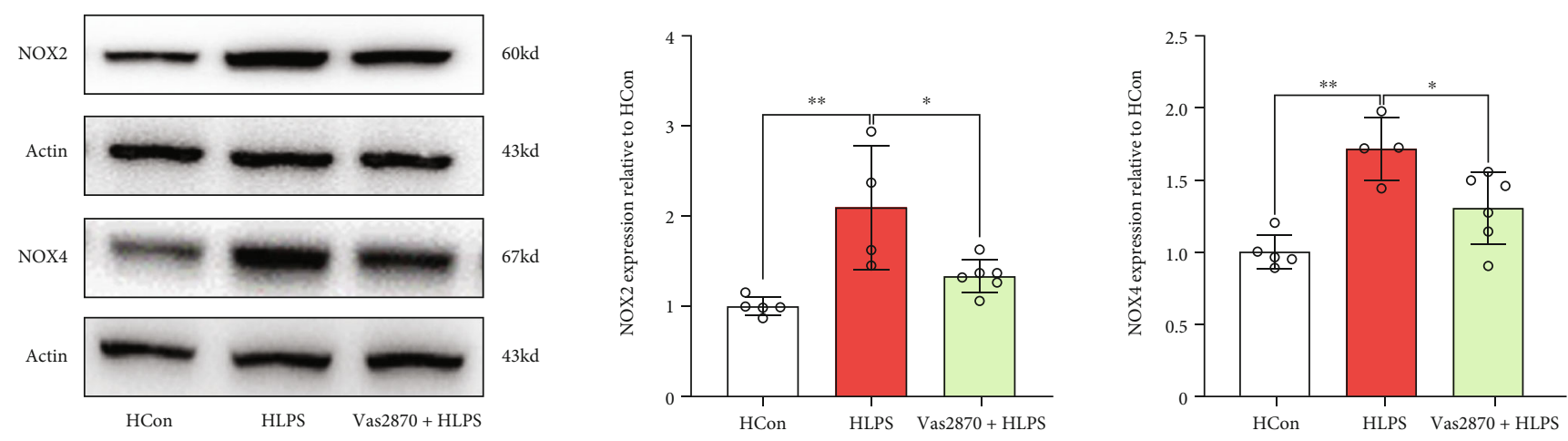

(n)

Figure 8: Inhibition of the NADPH oxidase pathway reduced septic renal injury via suppressing renal oxidative stress in diabetic mice. The survival rate (a) of the mice was calculated. Renal pathology was clearly observed via renal H\&E ((b) 200x) staining and PAS staining ((c) 200x). Serum BUN (d), CCr (e), and Cysc (f) levels were determined. MDA was measured using ELISA (g). Renal 4-hydroxynonenal (4HNE) immunohistochemical staining (h), renal ROS immunofluorescence staining (i), and 8-hydroxydeoxyguanosine (8-OHdG) immunohistochemical staining (j) were performed. Semiquantitative analysis of 4-HNE immunohistochemical staining (k), ROS immunofluorescence staining (l), and 8-OHdG immunohistochemical staining (m). Renal NADPH subunits NOX2 and NOX4 were detected by western blotting $(n)$. Each bar represents the mean \pm SEM $(n=4-10)$. ${ }^{*} p<0.05$ and ${ }^{* *} p<0.01$, one-way ANOVA with Tukey's test. Hcon: mice fed a HDF diet for 12 weeks received the same volume of solvent without LPS; HLPS: mice fed a HDF diet for 12 weeks and subjected to LPS (10 mg/kg, intraperitoneally) stimulation; Vas2870+HLPS: Vas2870 (10 mg/kg) was administered intraperitoneally $3 \mathrm{~h}$ before LPS administration.

function and oxidative stress in mice subjected to LPS stimulation, which led to a higher mortality rate compared to that of chow-fed mice. Septic renal injury was attenuated by a ferroptosis inhibitor in HFD-challenged mice. Furthermore, ferroptosis accumulation, which was altered by LPS stimulation in HFD-challenged mice, was suppressed by the NADPH oxidase inhibitor, Vas2870, in vivo. Our results indicated that HFD-induced diabetes may act as a potential risk factor that aggravates renal injury, particularly under critical conditions such as sepsis.

Ferroptosis is a type of iron-dependent programmed cell necrosis, which is mainly caused by ROS accumulation and characterized by extensive lipid peroxidation and subsequent severe mitochondrial and cell membrane dysfunction $[21,22]$. During AKI, renal tubular epithelial cell necroptosis [23], necrosis [24], and apoptosis [25] have been identified as the predominant types of cell death that lead to irreversible renal dysfunction. However, a recent study showed that ferroptosis is part of an adaptive response that unsuccessfully attempts to restore renal cellular homeostasis [26]; hence, pharmacological intervention is required to restore renal function [26], without inducing tubular epithelial cell necrosis or apoptosis. In the present study, ferroptosis inhibitor Fer-1 intervention reversed sepsis-associated AKI, which was aggravated in HFD-challenged mice. Our results indicate that it is critical to block ferroptosis formation in the early stage of sepsis-associated AKI, which may restore renal function by rebalancing renal cellular homeostasis. Our findings were consistent with those of Martin-Sanchez et al., in which Fer-1 could preserve renal function from nephrotoxic folic acid-induced AKI [27].

Notably, we found that ferroptosis was increased in HFD-challenged mice subjected to LPS stimulation. To date, studies have reported that ferroptosis can be activated in diabetes [28] and LPS stimulation [29]. However, in the present study, we found that renal ferroptosis elicited no significant effect in HFD-challenged mice without LPS stimulation. Therefore, in the process of sepsis-associated AKI in HFDchallenged mice, LPS stimulation initially induces ferroptosis, while the diabetes state may slow down ROS clearance and ferroptosis elimination, which together deteriorates renal function. Inhibition of ROS release may effectively reduce ferroptosis formation $[30,31]$. NADPH oxidase is the main source of ROS production in patients with diabetes [32] and sepsis [33]. In the present study, Vas2870 effectively reduced ROS release and subsequently attenuated renal ferroptosis formation, resulting in the restoration of renal function following LPS stimulation in HFD-challenged mice. These results indicate that inhibition of NADPH oxidase may either reduce ROS release from LPS stimulation or decrease oxidative stress in the diabetic state, which synergistically attenuates ferroptosis formation and improves renal function.

Notably, in the current study, we only observed the change of mitochondria in renal tubular epithelial cells, although there are several other cell types including Sertoli, glomerular vascular endothelial, and immune cells that contribute to sepsis-associated AKI; therefore, the specific cell type undergoing ferroptosis during sepsis-associated AKI requires further investigation. And more molecular mechanisms will be found in our further study. Moreover, the NADPH oxidase pathway in addition to the mitochondrial enzymes associated with the respiratory chain promotes extracellular ROS generation. However, potential renalprotective effects of mitochondrial enzymes associated with the respiratory chain require further investigation.

\section{Conclusion}

HFD-induced diabetes exacerbates sepsis-associated AKI via upregulation of NADPH oxidase, subsequent ROS release, 

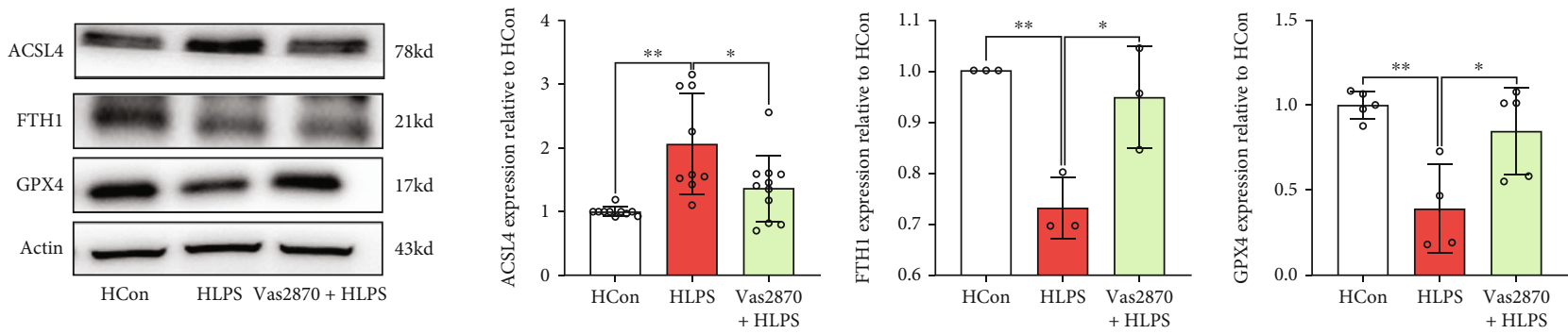

(a)

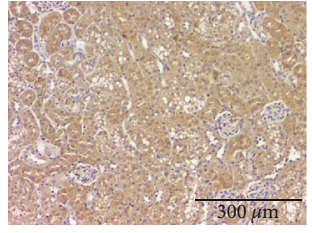

HCon

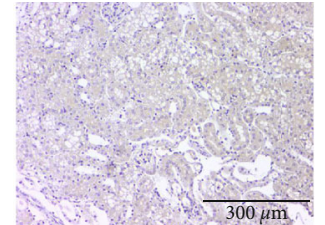

HLPS

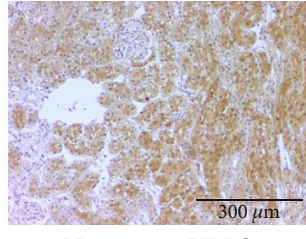

Vas2870 + HLPS

(b)
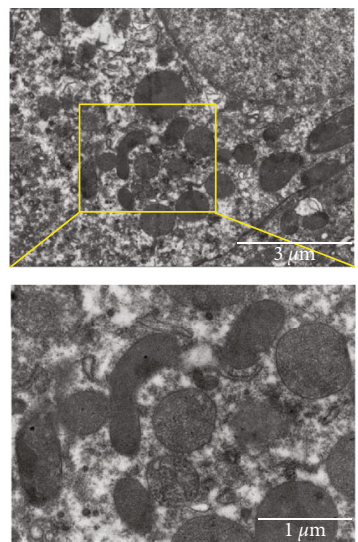

HLPS

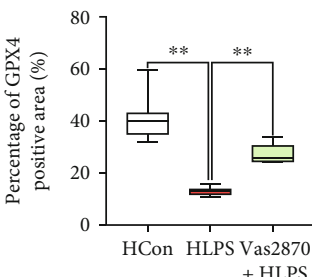

+ HLPS
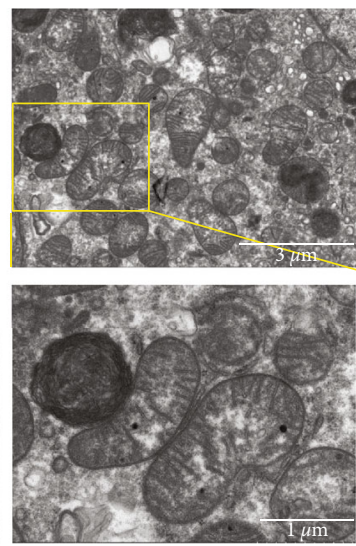

Vas 2870 + HLPS

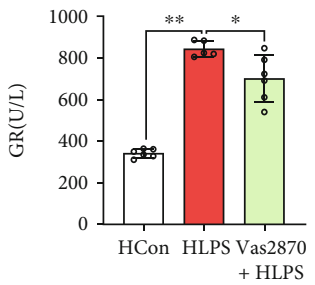

(c)

(d)

FIGURE 9: Inhibition of the NADPH oxidase pathway attenuated renal ferroptosis accumulation. Ferroptosis-related proteins, including ASCL4, FTH1, and GPX4, were determined by western blotting (a). GPX4 levels were also detected by immunohistochemical staining (b). The renal scavenging effect on ROS is reflected by the glutathione reductase (GR) level (c). Morphological features of mitochondria were measured using electron microscopy (d). Each bar represents the mean \pm SEM $(n=3-8)$. ${ }^{*} p<0.05$ and ${ }^{* *} p<0.01$, one-way ANOVA with Tukey's test.

and ferroptosis accumulation. NADPH inhibition could restore septic renal function from injury by suppressing ferroptosis accumulation in HFD-challenged mice, suggesting that targeting NADPH-mediated ROS release and ferroptosis accumulation is a novel therapeutic strategy to protect the kidney from septic injury in patients with obesity and type $2 \mathrm{DM}$.

\section{Abbreviations}

HDF: High-fat diet

Fer-1: Ferrostatin-1

ROS: Reactive oxygen species

AKI: Acute kidney injury

NOXs: Nicotinamide adenine dinucleotide phosphate oxidase
DM: Diabetes mellitus

OGTT: Oral gavage glucose tolerance test

ipITT: Intraperitoneal insulin tolerance test

BUN: Serum urea nitrogen

CCr: Creatinine

GR: Glutathione reductase

Cysc: Cystatin C

MDA: Malondialdehyde

TEM: Transmission electron microscopy

HE: Hematoxylin-eosin

PAS: Periodic acid-Schiff.

\section{Data Availability}

The data that support the findings of this study are available from the corresponding author upon reasonable request. 


\section{Conflicts of Interest}

The authors declare that they have no competing interests.

\section{Authors' Contributions}

Weifeng Yao, Haofeng Liao, and Mengya Pang contributed equally to this work. Mian Ge and Chenfang Luo conceived and designed the experiments. Weifeng Yao, Haofeng Liao, Mengya Pang, Lijie Pan, Yu Guan, and Xiaolei Huang performed the experiments. Haofeng Liao analyzed the data. Ziqing Hei contributed reagents, materials, and analysis tools. Xiaolei Huang contributed to the revised of manuscript. Mian Ge and Weifeng Yao wrote the paper. All authors read and approved the manuscript.

\section{Acknowledgments}

We are thankful to Lianxiong Yuan (Department of Scientific Research) at the Third Affiliated Hospital of Sun Yatsen University for providing statistical analysis tools. This study is supported by the National Natural Science Foundation of China (Beijing, China; Grant No. 81970546 for Mian Ge), Natural Science Foundation of Guangdong Province (Guangzhou, Guangdong Province, China; Grant No. 2020A1515010147 for Mian Ge), Guangdong Basic and Applied Basic Research Foundation (No. 2019A1515011766 and 2021A1515010255 for Chenfang Luo), Shenzhen Science, Technology and Innovation Commission (SZSTI) (No. JCYJ20180306173050592 for Xiaolei Huang), and National Natural Science Foundation of China (No. 81974081 for Weifeng Yao).

\section{Supplementary Materials}

Supplementary Figure 1: Fer-1 did not affect the protein expression of NOX in LPS-tread diabetic mice. The expression levels of NOX2 and NOX4 proteins were determined by western blotting (A). Each bar represents the mean \pm SEM $(n=4)$. $n s$ means $p>0.05$, T-test. (Supplementary Materials)

\section{References}

[1] N. Sarwar, P. Gao, S. R. Seshasai et al., "Diabetes mellitus, fasting blood glucose concentration, and risk of vascular disease: a collaborative meta-analysis of 102 prospective studies," Lancet, vol. 375, no. 9733, pp. 2215-2222, 2010.

[2] C. Fleischmann, A. Scherag, N. K. Adhikari et al., "Assessment of global incidence and mortality of hospital-treated sepsis. Current estimates and limitations," American Journal of Respiratory and Critical Care Medicine, vol. 193, no. 3, pp. 259-272, 2016.

[3] L. A. van Vught, B. P. Scicluna, A. J. Hoogendijk et al., "Association of diabetes and diabetes treatment with the host response in critically ill sepsis patients," Critical Care, vol. 20, no. 1, p. 252, 2016.

[4] P. Schuetz, P. Castro, and N. I. Shapiro, "Diabetes and sepsis: preclinical findings and clinical relevance," Diabetes Care, vol. 34, no. 3, pp. 771-778, 2011.
[5] S. Ma, R. G. Evans, N. Iguchi et al., "Sepsis-induced acute kidney injury: a disease of the microcirculation," Microcirculation, vol. 26, no. 2, article e12483, 2019.

[6] A. M. Esper, M. Moss, and G. S. Martin, "The effect of diabetes mellitus on organ dysfunction with sepsis: an epidemiological study," Critical Care, vol. 13, no. 1, p. R18, 2009.

[7] B. R. Shah and J. E. Hux, "Quantifying the risk of infectious diseases for people with diabetes," Diabetes Care, vol. 26, no. 2, pp. 510-513, 2003.

[8] R. Alobaidi, R. K. Basu, S. L. Goldstein, and S. M. Bagshaw, "Sepsis-associated acute kidney injury," Seminars in Nephrology, vol. 35, no. 1, pp. 2-11, 2015.

[9] S. C. Trevelin, D. Carlos, M. Beretta, J. S. da Silva, and F. Q. Cunha, "Diabetes mellitus and sepsis," Shock, vol. 47, no. 3, pp. 276-287, 2017.

[10] D. R. Emlet, A. D. Shaw, and J. A. Kellum, "Sepsis-associated AKI: epithelial cell dysfunction," Seminars in Nephrology, vol. 35, no. 1, pp. 85-95, 2015.

[11] J. M. Dennis and P. K. Witting, "Protective role for antioxidants in acute kidney disease," Nutrients, vol. 9, no. 7, p. 718, 2017.

[12] S. N. Heyman, S. Rosen, and C. Rosenberger, "A role for oxidative stress," Contributions to Nephrology, vol. 174, pp. 138-148, 2011.

[13] F. Magnani and A. Mattevi, "Structure and mechanisms of ROS generation by NADPH oxidases," Current Opinion in Structural Biology, vol. 59, pp. 91-97, 2019.

[14] K. M. Ralto, E. P. Rhee, and S. M. Parikh, "NAD(+) homeostasis in renal health and disease," Nature Reviews Nephrology, vol. 16, no. 2, pp. 99-111, 2020.

[15] Q. Yang, L. Gao, X. W. Hu et al., "Smad3-Targeted therapy protects against cisplatin-induced AKI by attenuating programmed cell death and inflammation via a NOX4dependent mechanism," Kidney Diseases, vol. 7, no. 5, pp. 372-390, 2021.

[16] X. M. Meng, G. L. Ren, L. Gao et al., "NADPH oxidase 4 promotes cisplatin-induced acute kidney injury via ROS-mediated programmed cell death and inflammation," Laboratory Investigation, vol. 98, no. 1, pp. 63-78, 2018.

[17] Z. X. Wang, J. Ma, X. Y. Li et al., "Quercetin induces p53independent cancer cell death through lysosome activation by the transcription factor EB and reactive oxygen speciesdependent ferroptosis," British Journal of Pharmacology, vol. 178, no. 5, pp. 1133-1148, 2021.

[18] S. F. Liou, T. T. N. Nguyen, J. H. Hsu et al., "The preventive effects of xanthohumol on vascular calcification induced by vitamin D3 plus nicotine," Antioxidants, vol. 9, no. 10, p. 956, 2020.

[19] J. B. Kornum, R. W. Thomsen, A. Riis, H. H. Lervang, H. C. Schonheyder, and H. T. Sorensen, "Type 2 diabetes and pneumonia outcomes," Diabetes Care, vol. 30, no. 9, pp. 22512257, 2007.

[20] V. Kaplan, D. C. Angus, M. F. Griffin, G. Clermont, R. Scott Watson, and W. T. Linde-Zwirble, "Hospitalized communityacquired pneumonia in the elderly," American Journal of Respiratory and Critical Care Medicine, vol. 165, no. 6, pp. 766-772, 2002.

[21] H. Yuan, J. Pratte, and C. Giardina, "Ferroptosis and its potential as a therapeutic target," Biochemical Pharmacology, vol. 186, article 114486, 2021.

[22] H. F. Yan, T. Zou, Q. Z. Tuo et al., "Ferroptosis: mechanisms and links with diseases," Signal Transduction and Targeted Therapy, vol. 6, no. 1, p. 49, 2021. 
[23] W. Jun, J. Benjanuwattra, S. C. Chattipakorn, and N. Chattipakorn, "Necroptosis in renal ischemia/reperfusion injury: a major mode of cell death?," Archives of Biochemistry and Biophysics, vol. 689, article 108433, 2020.

[24] A. Pefanis, F. L. Ierino, J. M. Murphy, and P. J. Cowan, "Regulated necrosis in kidney ischemia-reperfusion injury," Kidney International, vol. 96, no. 2, pp. 291-301, 2019.

[25] G. Priante, L. Gianesello, M. Ceol, D. Del Prete, and F. Anglani, "Cell death in the kidney," International Journal of Molecular Sciences, vol. 20, no. 14, p. 3598, 2019.

[26] C. Chen, D. Wang, Y. Yu et al., "Legumain promotes tubular ferroptosis by facilitating chaperone-mediated autophagy of GPX4 in AKI," Cell Death \& Disease, vol. 12, no. 1, p. 65, 2021.

[27] D. Martin-Sanchez, O. Ruiz-Andres, J. Poveda et al., "Ferroptosis, but not necroptosis, is important in nephrotoxic folic acid-induced AKI," Journal of the American Society of Nephrology, vol. 28, no. 1, pp. 218-229, 2017.

[28] X. Feng, S. Wang, Z. Sun et al., "Ferroptosis enhanced diabetic renal tubular injury via HIF- $1 \alpha / \mathrm{HO}-1$ pathway in $\mathrm{db} / \mathrm{db}$ mice," Frontiers in Endocrinology, vol. 12, article 626390, 2021.

[29] B. M. Oh, S. J. Lee, G. L. Park et al., "Erastin inhibits septic shock and inflammatory gene expression via suppression of the NF- $\kappa$ B pathway," Journal of Clinical Medicine, vol. 8, no. 12, p. 2210, 2019.

[30] D. Tang, X. Chen, R. Kang, and G. Kroemer, "Ferroptosis: molecular mechanisms and health implications," Cell Research, vol. 31, no. 2, pp. 107-125, 2021.

[31] F. Kuang, J. Liu, D. Tang, and R. Kang, "Oxidative damage and antioxidant defense in ferroptosis," Frontiers in Cell and Development Biology, vol. 8, article 586578, 2020.

[32] Q. Yang, F. R. Wu, J. N. Wang et al., "Nox4 in renal diseases: an update," Free Radical Biology \& Medicine, vol. 124, pp. 466-472, 2018.

[33] X. Kong, R. Thimmulappa, P. Kombairaju, and S. Biswal, "NADPH oxidase-dependent reactive oxygen species mediate amplified TLR4 signaling and sepsis-induced mortality in Nrf2-deficient mice," Journal of Immunology, vol. 185, no. 1, pp. 569-577, 2010. 